\section{Return to Driving and a Clinical Measure of Reaction Time}

To the Editor,

We read the informative and clinically relevant "Point/ Counterpoint" discussion by Drs. Finestone and Gershkoff in the September 2011 issue of PM\&R with great interest [1]. We wish to comment on a portion of Dr. Gershkoff's initial response, in which he states that "Other possible evaluation tools include driver simulators and other computerized assessment tools for vision, perception, and reaction time" (p. 877). This statement is not inaccurate but may lead the reader to believe that the use of a computer with dedicated software is the only reliable method for measuring reaction time (RT). Simple RT, a measure of how quickly a person responds in the same manner to a specific stimulus, powerfully influences function and health. As the authors undoubtedly knew, a prolonged RT increases risk for motor vehicle accidents and accidental falls. Less obvious, however, are known relationships between RT and cognition, and even mortality, which add to the relevance of this measure of human function.

In an effort to make the determination of RT available to physiatrists and other health care practitioners, we developed a clinical method of determining $\mathrm{RT}\left(\mathrm{RT}_{\text {clin }}\right)$. $\mathrm{RT}_{\text {clin }}$ measures the time required to catch a suspended vertical shaft by hand closure. The device, equipped with a spacer at the lower end to standardize finger closure distance, is released by the examiner at random intervals through the subject's open hand. The subject grasps the apparatus as quickly as possible as soon as it is perceived to be falling. The distance the device falls is measured, and the elapsed time, $\mathrm{RT}_{\text {clin, }}$, is calculated by use of the acceleration of gravity. (A figure depicting the device is available in $\mathrm{PM} \& \mathrm{R}$ [2].)

We found this simple, inexpensive technique to be reliable over a wide range of re-test times and to be valid as determined by age effect, response to a competing task, and direct comparisons with accepted computerized techniques [3-5]. In addition, $\mathrm{RT}_{\text {clin }}$ correlates strongly with an important sport-related measure of function, the ability to quickly protect the face with the hands from an onrushing projectile [6]. Finally, the technique appears to be sensitive to the effects of sport-related concussion [2] and demonstrates strong correlations with quantified sensorimotor functions of the lower limbs [7].

Although more research is needed to identify precise relationships between $\mathrm{RT}_{\text {clin }}$ and specific driving skills, sufficient work has accumulated to suggest that physiatrists and other health care practitioners do indeed have a simple, inexpensive method for measuring RT in the clinic. Furthermore, this method has been found to be valid compared with computerized techniques, to demonstrate good inter-rater and re-test reliabilities, and to correlate strongly with a complex upper limb task involving self-protection from a flying projectile. With regard to this strong correlation, no computerized technique can make a similar claim.

It is hoped that physiatrists and other health care practitioners will increasingly recognize the functional relevance of RT and, furthermore, realize that a computer and dedicated software are not necessary for its measurement. Moreover, we look forward to the time when the relationships between relevant driving reaction times and $\mathrm{RT}_{\text {clin }}$ are known so the technique can allow physiatrists to offer a more objective and informed opinion with regard to return to driving, an issue of importance to the patient and society as a whole.

James K. Richardson, MD James T. Eckner, MD

Department of Physical Medicine and Rehabilitation University of Michigan Hospital and Health Systems

Ann Arbor, MI

J.K.R. Disclosure: 4A, University of Michigan patent application for a reaction time device related to the one described in the letter; 8A, ROI-NIN 2008-2013

JTE: Disclosure: 4A, University of Michigan patent application for a reaction time device related to the one described in the letter; 8A, K-12/RMSTP grant and pilot grant from Univ Michigan SHARP Center

DOI: 10.1016/j.pmrj.2011.12.003

\section{REFERENCES}

1. Finestone HM, Gershkoff A, Watanabe T. Driving after stroke: What are the appropriate criteria? PM R 2011;3:873-878.

2. Eckner JT, Kutcher J, Richardson JK. Effect of concussion on clinically measured reaction time in nine NCAA Division I collegiate athletes: A preliminary study. PM R 2011;3:212-218.

3. Eckner JT, Whitacre RD, Kirsch NL, Richardson JK. Evaluating a clinical measure of reaction time: An observational study. Percept Mot Skills 2009;108:717-720.

4. Eckner JT, Kutcher JS, Richardson JK. Pilot evaluation of a novel clinical test of reaction time in National Collegiate Athletic Association Division I football players. J Athl Train 2010;45:327-332.

5. Eckner JT, Kutcher J, Richardson JK. Between season test-retest reliability of clinically measured reaction time in NCAA Division I collegiate athletes. J Athl Train 2010;45:409-414.

6. Eckner JT, Kim H, Lipps DB, Richardson JK, Ashton-Miller JA. Can a clinical measure of reaction time predict a functional sport-protective response? Med Sci Sports Exerc 2011;43:382-387.

7. Richardson JK, Gimpert R, Kim H, et al. Is a clinical measure of upper limb reaction time predictive of lower limb neuromuscular function? Abstract, AAPM\&R Annual Meeting, Orlando, FL, November 2011.

\section{REPLY:}

Drs. Richardson and Eckner are correct in pointing out that noncomputerized electronic and mechanical devices to measure reaction time (RT) do exist (Dennis Magrann, personal communication, OTR CDRS, MossRehab Driving 